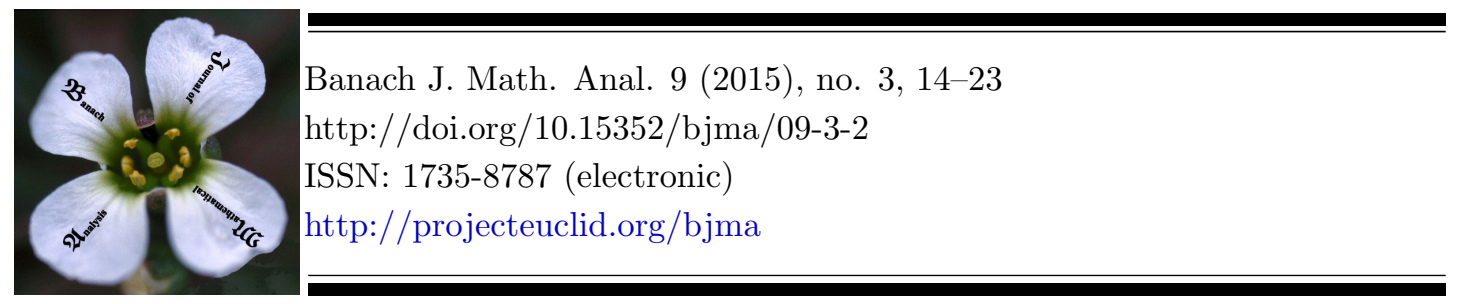

\title{
ON POSITIVE DEFINITE DISTRIBUTIONS WITH COMPACT SUPPORT
}

\author{
SAULIUS NORVIDAS
}

Communicated by J. A. Ball

\begin{abstract}
We propose necessary and sufficient conditions for a distribution (generalized function) $f$ of several variables to be positive definite. For this purpose, certain analytic extensions of $f$ to tubular domains in complex space $\mathbb{C}^{n}$ are studied. The main result is given in terms of completely monotonic functions on convex cones in $\mathbb{R}^{n}$.
\end{abstract}

\section{INTRODUCTION}

Let $\mathbb{R}^{n}$ be the real $n$-dimensional Euclidean space imbedded in $\mathbb{C}^{n}$ so that $\mathbb{C}^{n}=\mathbb{R}^{n}+i \mathbb{R}^{n}$. For $u \in \mathbb{R}^{n}$, we set $|u|=\left|u_{1}\right|+\cdots+\left|u_{n}\right|$. Let $|x|_{2}$ denote the standard Euclidean norm on $\mathbb{R}^{n}$. If, in addition, the entries of $u$ are nonnegative integers, then we call $u$, throughout the following, a multi-index.

The space of test functions $\mathcal{E}\left(\mathbb{R}^{n}\right)$ is the set of $\varphi: \mathbb{R}^{n} \rightarrow \mathbb{C}$ such that $D_{x}^{u} \varphi$ is continuous for all multi-indices $u$. Here $D_{x}^{u}=D_{x_{1}}^{u_{1}} \cdots D_{x_{n}}^{u_{n}}$ and $D_{x_{k}}=\partial / \partial x_{k}$. Let $D\left(\mathbb{R}^{n}\right)$ denote the subspace of $\mathcal{E}\left(\mathbb{R}^{n}\right)$ consisting of functions with compact support. We assume that the topologies on $\mathcal{E}\left(\mathbb{R}^{n}\right)$ and on $D\left(\mathbb{R}^{n}\right)$ are introduced as usual (see, e.g., [7] or [16]). The elements of the conjugate spaces $\mathcal{E}^{\prime}\left(\mathbb{R}^{n}\right)$ and $D^{\prime}\left(\mathbb{R}^{n}\right)$ are called distributions or generalized functions. Since $D\left(\mathbb{R}^{n}\right)$ is continuously imbedded in $\mathcal{E}\left(\mathbb{R}^{n}\right)$, it follows that each $f \in D^{\prime}\left(\mathbb{R}^{n}\right)$ gives an element of $\mathcal{E}^{\prime}\left(\mathbb{R}^{n}\right)$ by restriction. Moreover, $\mathcal{E}^{\prime}\left(\mathbb{R}^{n}\right)$ coincides with a subspace of compactly supported distributions of $D^{\prime}\left(\mathbb{R}^{n}\right)$.

Date: Received: May 14, 2014; Accepted: Sep. 16, 2014.

2010 Mathematics Subject Classification. Primary 46F20; Secondary 42B10.

Key words and phrases. Positive definite distributions, analytic representations of distributions, Cauchy transform, completely monotonic functions, convex cones. 
If $f \in D^{\prime}\left(\mathbb{R}^{n}\right)$, then the action of $f$ on $\omega \in D\left(\mathbb{R}^{n}\right)$ is written as $(f, \omega)$. A distribution $f \in D^{\prime}\left(\mathbb{R}^{n}\right)$ is said to be positive definite if for all $\varphi \in D\left(\mathbb{R}^{n}\right)$,

$$
(f, \varphi * \widetilde{\varphi}) \geq 0
$$

were $\widetilde{\varphi}(x):=\overline{\varphi(-x)}$, and $*$ denotes the usual convolution operator. The BochnerSchwartz theorem gives a representation of a positive definite distribution in terms of the Fourier transform. Let us recall some notion.

The Schwartz class $S\left(\mathbb{R}^{n}\right)$ can be defined as the set of $\omega \in \mathcal{E}\left(\mathbb{R}^{n}\right)$ satisfying

$$
\|\omega\|_{m}:=\sup _{x \in \mathbb{R}^{n},|u| \leq m}\left|\left(1+|x|_{2}\right)^{m} D_{x}^{u} \omega(x)\right|<\infty
$$

for all nonnegative integers $m$. Semi-norms (1.2) turns $S\left(\mathbb{R}^{n}\right)$ into a Fréchet space. The elements of $S^{\prime}\left(\mathbb{R}^{n}\right)$ are called tempered distributions. For $\varphi \in S\left(\mathbb{R}^{n}\right)$, we define the Fourier transform as

$$
\widehat{\varphi}(x)=\int_{\mathbb{R}^{n}} e^{i(x, t)} \varphi(t) d t, \quad x \in \mathbb{R}^{n}
$$

where $(x, t)=x_{1} t_{1}+\cdots+x_{n} t_{n}$. If $f \in S^{\prime}\left(\mathbb{R}^{n}\right)$, then the Fourier transform $\mathcal{F}[f]$ can be defined by

$$
(\mathcal{F}[f], \varphi)=(f, \widehat{\varphi}), \quad \varphi \in S\left(\mathbb{R}^{n}\right) .
$$

The Bochner-Schwartz theorem states (see, e.g., [16, p. 125]) that $f \in D^{\prime}\left(\mathbb{R}^{n}\right)$ is positive definite if and only if there exists nonnegative tempered measure $\eta \in$ $S^{\prime}\left(\mathbb{R}^{n}\right)$ such that $f=\mathcal{F}[\eta]$. We recall that a nonnegative measure $\eta$ is said to be tempered if there exists $\alpha \geq 0$ such that

$$
\int_{\mathbb{R}^{n}}\left(1+|x|_{2}\right)^{-\alpha} d \eta<\infty
$$

Note that this theorem implies that any positive definite distribution belongs to $S^{\prime}\left(\mathbb{R}^{n}\right)$.

There are many other than the Bochner-Schwartz theorem characterizations of positive definite functions (see, e.g., [8, p.p. 70-83]). As far as we known, it is perhaps surprising that there are almost no such results for distributions. We mention only [13], where attention has been paid to positive definite distributions of order zero on $\mathbb{R}$, with applications to a Volterra equation. See also survey article [11]. Note also that in [4] the Bochner-Schwartz theorem was generalized for the spaces of Fourier hyperfunctions and hyperfunctions.

In this paper, we wish to explore the idea of how to describe positive definite $f \in S^{\prime}\left(\mathbb{R}^{n}\right)$ by means of its analytic representations in $\mathbb{C}^{n}$. Let us start with the case of one variable.

For $f \in D^{\prime}(\mathbb{R})$, Tillman [15] has proved that there exists a pair of functions $f^{(+)}$and $f^{(-)}$, analytic in the open upper $\mathbb{C}^{(+)}$and in the open lower half-plane $\mathbb{C}^{(-)}$, respectively, such that

$$
\lim _{\varepsilon \rightarrow+0} \int_{-\infty}^{\infty}\left(f^{(+)}(t+i \varepsilon)-f^{(-)}(t-i \varepsilon)\right) \varphi(t) d t=(f, \varphi)
$$


for all $\varphi \in D(\mathbb{R})$. This pair $\left(f^{(+)}, f^{(-)}\right)$(or sectionally analytic function on $\left.\mathbb{C}^{(+)} \cup \mathbb{C}^{(-)}=\mathbb{C} \backslash \mathbb{R}\right)$ is called an analytic representation of $f$.

If a distribution $f$ has a compact support, then an analytic representation of $f$ can be obtained using an explicit construction. Indeed, if $f \in \mathcal{E}^{\prime}(\mathbb{R})$, then

$$
K(f)(z)=\frac{1}{2 \pi i}\left(f(\cdot),(\cdot-z)^{-1}\right)=\frac{1}{2 \pi i}\left(f_{t},(t-z)^{-1}\right)
$$

is well defined for all $z \in \mathbb{C} \backslash \mathbb{R}$. The function $K(f)$ is called the Cauchy transform of $f$. If we take $\left(f^{(+)}, f^{(-)}\right)=K(f)$, then we obtain an analytic representation of $f$ (see, e.g., [1, p. 155]). Note that analytic representations of the same distribution differ by at most an entire function [1].

For any fixed $z \in \mathbb{C} \backslash \mathbb{R}$, the Cauchy kernel $k(t)=(t-z)^{-1}$ belongs to $\mathcal{E}(\mathbb{R})$ but not to $S(\mathbb{R})$. Hence, for all $f \in S^{\prime}(\mathbb{R})$, the analytic representation (1.5), in general, does not exists (see details in [1, p. 156]). On the other hand, if $f \in S^{\prime}(\mathbb{R})$, then there exists a nonnegative integer $m_{0}$ such that $f$ is continuous in the semi-norm $\|\cdot\|_{m_{0}}$ defined in (1.2), i.e., there exist $A>0$ such that $|(f, \varphi)| \leq A\|\varphi\|_{m_{0}}$ for all $\varphi \in S(\mathbb{R})$ (see $[16$, p. 74$]$ ). We call the smallest $m_{0}$ that satisfies the above inequality the $S$-order of $f \in S^{\prime}(\mathbb{R})$. Let us write $\varrho_{S}(f)$ for this order. Note that $\varrho_{S}(f)$ is different from the usual order $\varrho_{D}(f)$ of $f$ as distribution in $D^{\prime}(\mathbb{R})$. Let us define the generalized Cauchy kernel $\widetilde{k}_{m}(t)$ to be $(t-z)^{-(m+1)}$. Now, if $f \in S^{\prime}(\mathbb{R})$ and $m$ is a nonnegative integer such that $m \geq \varrho_{S}(f)$, then $\left(f_{t}, \widetilde{k}_{m}(t)\right)$ is well defined. We derived in [9] necessary and sufficient conditions for $f \in S^{\prime}(\mathbb{R})$ to be a positive definite distribution in terms of this transform. Let us recall that a function $\theta:(a, b) \rightarrow \mathbb{R},-\infty \leq a<b \leq \infty$, is said to be completely monotonic if it is infinitely differentiable and $(-1)^{n} \theta^{(n)}(y) \geq 0$ for each $y \in(a, b)$ and all $n=0,1,2, \ldots$ Further, $\theta(y)$ is said to be absolutely monotonic on $(a, b)$ if a $\theta(-y)$ is completely monotonic on $(-b,-a)$.

Theorem 1.1. ([9], Theorem 1.3) Let $f \in S^{\prime}(\mathbb{R})$ and let $n$ be an integer such that $2 n \geq \varrho_{f}$. Suppose that $a_{1}, a_{2} \in \mathbb{R}, a_{1} \neq a_{2}$. Let

$$
\widetilde{K}(f, j)(z)=(-1)^{n} \frac{i}{\pi}\left(e^{i a_{j} t} f_{t},(z-t)^{-(2 n+1)}\right),
$$

$z \in \mathbb{C} \backslash \mathbb{R}, j=1,2$. Then $f$ is positive definite if and only if:

(i) $y \rightarrow \widetilde{K}(f, j)(i y), j=1,2$ are completely monotonic functions for $y \in$ $(0, \infty)$;

(ii) $y \rightarrow-\widetilde{K}(f, j(i y), j=1,2$ are absolutely monotonic functions for $y \in$ $(-\infty, 0)$.

It is quite possible that similar results are also valid for $f \in S^{\prime}\left(\mathbb{R}^{n}\right)$. Of course, for $n>1$, it is natural to use the Cauchy kernel $K_{\Gamma}$ with respect to a cone $\Gamma$ in $\mathbb{R}^{n}$ (see its definition (1.6)). Then the Cauchy transform $K_{\Gamma}(f)$ of $f$ is defined as the convolution of $K_{\Gamma}$ with $f$. Note that the case of several variables is much more difficult than the one-dimensional case. At first, we do not fully understand how to define the generalized Cauchy kernel $\widetilde{K_{\Gamma}}$ to get well defined transform $f * \widetilde{K_{\Gamma}}$ on $S^{\prime}\left(\mathbb{R}^{n}\right)$. Second, the process of taking boundary values as in (1.4) are investigated only for some proper subclasses of $S^{\prime}\left(\mathbb{R}^{n}\right)$ (see, e.g., [3]). Finally, we note that 
the main purpose of this paper is to provide a criterion for a distribution to be positive definite. Therefore, to simplify the technical details, we obtain here a criterion only for compactly supported distributions.

A set $\Gamma \subset \mathbb{R}^{n}$ is said to be a cone if $x \in \Gamma$ implies $\alpha x \in \Gamma$ for all $\alpha>0$. The dual cone of $\Gamma$ is defined by

$$
\Gamma^{*}=\left\{t \in \mathbb{R}^{n}:(x, t) \geq 0 \text { for all } x \in \Gamma\right\} .
$$

The cone $\Gamma^{*}$ is always closed convex, and $\left(\Gamma^{*}\right)^{*}=\overline{\operatorname{ch} \Gamma}$, where $\operatorname{ch} \Gamma$ denotes the convex hull of $\Gamma$. Next, $\Gamma$ is called salient (acute) if $\overline{c h} \Gamma$ does not contain any straight line in $\mathbb{R}^{n}$. This is equivalent to $\operatorname{int}\left(\Gamma^{*}\right) \neq \emptyset$. A cone $\Gamma$ is said to be regular if $\Gamma$ is an open convex salient cone. if

Let $\left\{\Lambda_{j}\right\}_{1}^{m}$ be a family of regular cones. We say that $\left\{\Lambda_{j}\right\}_{1}^{m}$ covers exactly $\mathbb{R}^{n}$

$$
\overline{\cup_{k=1}^{m} \Lambda_{j}}=\mathbb{R}^{n}
$$

and the Lebesgue measure of $\overline{\Lambda_{i}} \cap \overline{\Lambda_{j}}$ is equal to zero whenever $i \neq j$. Any $\omega=\left(\omega_{1}, \ldots, \omega_{n}\right)$ whose entries $\omega_{k}$ are -1 or 1 defines the cone $Q_{\omega}=\left\{x \in \mathbb{R}^{n}\right.$ : $x_{k} \omega_{k}>0$ for $\left.k=1, \ldots, n\right\}$. The cone $Q_{\omega}$ is called a quadrant. The collection of all $2^{n}$ regular cones $\left\{Q_{\omega}\right\}_{\omega}$ covers exactly $\mathbb{R}^{n}$.

Let $\Gamma$ be an open cone in $\mathbb{R}^{n}$. Then $T_{\Gamma}=\mathbb{R}^{n}+i \Gamma=\left\{z=x+i y: x \in \mathbb{R}^{n}, y \in \Gamma\right\}$ is called a tube domain in $\mathbb{C}^{n}$. If $\Gamma$ is regular, then the Cauchy kernel of $\Gamma$ (or with respect to $\Gamma$ ) is defined as

$$
K_{\Gamma}(z)=\int_{\Gamma^{*}} e^{i(z, t)} d t, \quad z \in T_{\Gamma} .
$$

The kernel $K_{\Gamma}$ is an analytic function on $T_{\Gamma}[16, \mathrm{p} .143]$. If $f$ is a distribution on $\mathbb{R}^{n}$, then

$$
K_{\Gamma}(f)(z)=\frac{1}{(2 \pi)^{n}}\left(f(\cdot), K_{\Gamma}(z-\cdot)\right)=\frac{1}{(2 \pi)^{n}}\left(f_{t}, K_{\Gamma}(z-t)\right)
$$

is called the Cauchy transform of $f$. For example, in $\mathbb{R}$ there are only two regular cones $(-\infty, 0)$ and $(0, \infty)$. If $\Gamma=(0, \infty)$, then we see that $(1.7)$ coincides with $(1.5)$.

Suppose that $\Gamma$ is a regular cone in $\mathbb{R}^{n}$. The directional derivation of a function $\theta: \Gamma \rightarrow \mathbb{C}$ along $a=\left(a_{1}, \ldots, a_{n}\right) \in \Gamma$ is defined as usual: $D_{a} \theta(y)=\left(a_{1} D_{y 1}+\right.$ $\left.\cdots+a_{n} D_{y_{n}}\right) \theta(y)$. Then $\theta$ is called completely monotonic if

$$
(-1)^{k} D_{\gamma_{1}} D_{\gamma_{2}} \ldots D_{\gamma_{k}} \theta(y) \geq 0, \quad k=0,1, \ldots,
$$

for all $y \in \Gamma$ and all $\gamma_{1}, \ldots, \gamma_{k} \in \Gamma$ (see [6, p. 172]).

Now we are able to describe positive definite distributions in $\mathcal{E}^{\prime}\left(\mathbb{R}^{n}\right)$.

Theorem 1.2. Assume that $\left\{\Gamma_{k}\right\}_{k=1}^{m}$ is a set of regular cones such that $\left\{\Gamma_{k}^{*}\right\}_{1}^{m}$ covers exactly $\mathbb{R}^{n}$. A distribution $f \in \mathcal{E}^{\prime}\left(\mathbb{R}^{n}\right)$ is positive definite if and only if $y \rightarrow$ $K_{\Gamma_{k}}(f)(i y), y \in \Gamma_{k}$, is a completely monotonic function for each $k=1,2, \ldots, m$. 


\section{Preliminaries AND PRoOfs}

We define the inverse Fourier transform of a bounded measure $\mu$ on $\mathbb{R}^{n}$ as

$$
\check{\mu}(\xi)=\frac{1}{(2 \pi)^{n}} \int_{\mathbb{R}^{n}} e^{-i(\xi, t)} d \mu(t) .
$$

In the case if $\mu$ has a density $\varphi \in L^{1}\left(\mathbb{R}^{n}\right)$, then the inverse transform of $\varphi$ is defined similarly. In addition, the following inversion formula $\widehat{(\breve{\varphi})}=\varphi$ holds for suitable functions $\varphi$.

Suppose that $\Lambda$ is a convex salient cone in $\mathbb{R}^{n}$, and let $S^{\prime}(\Lambda)$ be the set of all $F \in S^{\prime}\left(\mathbb{R}^{n}\right)$ supported on $\Lambda$. For any fixed $y \in \mathbb{R}^{n}$, the Laplace transform of $F \in S^{\prime}(\Lambda)$ is defined by

$$
L_{y}(F)(x)=\mathcal{F}\left[F(\cdot) e^{-(y, \cdot)}\right](x)=\mathcal{F}_{\xi}\left[F(\xi) e^{-(y, \xi)}\right](x), \quad x \in \mathbb{R}^{n},
$$

where $\mathcal{F}: S^{\prime}\left(\mathbb{R}^{n}\right) \rightarrow S^{\prime}\left(\mathbb{R}^{n}\right)$ is the Fourier transform defined in (1.3). If $y \in \operatorname{int} \Lambda^{*}$, then $F(\cdot) e^{-(y, \cdot)}$ belongs to $S^{\prime}\left(\mathbb{R}^{n}\right)$ (see, e.g., [16, p. 127]). Hence, $L_{y}(F)(x)$ is well defined for all $x \in \mathbb{R}^{n}$. Further, $L_{y}(F)(x)$ is analytic on $T_{\operatorname{int} \Lambda^{*}}$ as a function of $z=x+i y$, and

$$
\frac{\partial^{|u|}}{\partial z_{1}^{u_{1}} \ldots \partial z_{n}^{u_{n}}} L_{y}(F)(x)=i^{|u|} \mathcal{F}_{\xi}\left[\left(\xi_{1}^{u_{1}} \cdots \xi_{n}^{u_{n}}\right) F(\xi) e^{-(y, \xi)}\right](x)
$$

for each multi-index $u=\left(u_{1}, \ldots, u_{n}\right)$ [16, p. 128].

Let $\chi_{A}$ denote the indicator function of $A \subset \mathbb{R}^{n}$. If we compare (1.6) and (2.1), then we have that

$$
\begin{gathered}
K_{\Gamma}(z)=\int_{\Gamma^{*}} e^{i(z, \xi)} d \xi=\int_{\mathbb{R}^{n}} \chi_{\Gamma^{*}}(\xi) e^{i(x, \xi)} e^{-(y, \xi)} d \xi=\mathcal{F}_{\xi}\left[\chi_{\Gamma^{*}}(\xi) e^{-(y, \xi)}\right](x) \\
=L_{y}\left(\chi_{\Gamma^{*}}\right)(x)
\end{gathered}
$$

for all $z=x+i y \in T_{\Gamma}$. This together with (1.7) and (2.2) implies the following lemma, where we collect certain facts about the Cauchy transform, which we need in this section. For a proof of this lemma we refer to [16, p.p. 144-145].

Lemma 2.1. Let $\Gamma$ be a regular cone in $\mathbb{R}^{n}$. The Cauchy kernel $K_{\Gamma}(z)$ is an analytic function for $z \in T_{\Gamma}=\mathbb{R}^{n}+i \Gamma$. If $f \in \mathcal{E}^{\prime}\left(\mathbb{R}^{n}\right)$, then the Cauchy transform (1.7) is well defined on $T_{\Gamma}$. Moreover, $K_{\Gamma}(f)$ is analytic on $T_{\Gamma}$ and

$$
\frac{\partial^{|u|}}{\partial z_{1}^{u_{1}} \ldots \partial z_{n}^{u_{n}}} K_{\Gamma}(f)(z)=\frac{1}{(2 \pi)^{n}}\left(f_{t}, \frac{\partial^{|u|}}{\partial z_{1}^{u_{1}} \ldots \partial z_{n}^{u_{n}}} K_{\Gamma}(z-t)\right)
$$

for all multi-index $u \in \mathbb{R}^{n}$.

Recall that a complex-valued function $u$ on $\mathbb{R}^{n}$ is said to be positive definite if

$$
\sum_{j, k=1}^{n} u\left(x_{j}-x_{k}\right) c_{j} \bar{c}_{k} \geq 0
$$

for any finite sets $x_{1}, \ldots, x_{n} \in \mathbb{R}^{n}$ and for any $c_{1}, \ldots, c_{n} \in \mathbb{C}$. The Bochner theorem (see, e.g., [2, p. 58], [5, p. 293] and [12, p.p. 41-47]) states that a continuous function $u: \mathbb{R}^{n} \rightarrow \mathbb{C}$ is positive definite if and only if it is the Fourier 
transform of a positive finite measure $\mu$ on $\mathbb{R}^{n}$. Note that if $u$ is continuous on $\mathbb{R}^{n}$, then the definition (2.4) is equivalent to

$$
\int_{\mathbb{R}^{n}} u(x)(\varphi * \widetilde{\varphi})(x) d x \geq 0,
$$

where $\varphi$ ranges over $L^{1}\left(\mathbb{R}^{n}\right)$ (or over all continuous functions on $\mathbb{R}^{n}$ with compact support). As usual, we identify a locally integrable function $u$ on $\mathbb{R}^{n}$ with a regular distribution by the formula

$$
(u, \varphi)=\int_{\mathbb{R}^{n}} u(x) \varphi(x) d x
$$

for suitable test functions. Of course, for regular distributions, both definitions (1.1) and (2.5) coincide. Note that any locally bounded measure $\mu$ on $\mathbb{R}^{n}$ also defines in a similar way as in (2.6) an integrable distribution.

We need a few simple facts about positive definite functions. The next lemmas are not new, but their proofs are added here for completeness.

Lemma 2.2. A distribution $f \in S^{\prime}\left(\mathbb{R}^{n}\right)$ is positive definite if and only if

$$
(f, \omega) \geq 0
$$

for all positive definite $\omega \in D\left(\mathbb{R}^{n}\right)$.

Proof. Assume that both $f \in S^{\prime}\left(\mathbb{R}^{n}\right)$ and $\omega \in D\left(\mathbb{R}^{n}\right)$ are positive definite. Using the Bochner theorem in $S\left(\mathbb{R}^{n}\right)$ for $\omega$, and the Bochner-Schwartz theorem in $S^{\prime}\left(\mathbb{R}^{n}\right)$ for $f$, we have that $\check{\omega}$ is a nonnegative function in $S\left(\mathbb{R}^{n}\right)$ and $\mathcal{F}[f]$ is a nonnegative tempered measure on $\mathbb{R}^{n}$. Then $(\mathcal{F}[f], \check{\omega})$ may be derived as the usual integral

$$
(\mathcal{F}[f], \check{\omega})=\int_{\mathbb{R}^{n}} \check{\omega}(x) d(\mathcal{F}[f](x)) .
$$

Moreover, $(\mathcal{F}[f], \check{\omega}) \geq 0$. Therefore, $(1.3)$ implies that $(f, \omega)=(\mathcal{F}[f], \check{\omega}) \geq 0$.

Let $f \in S^{\prime}\left(\mathbb{R}^{n}\right)$ and let $\varphi \in D\left(\mathbb{R}^{n}\right)$. The Fourier transform of $\varphi * \widetilde{\varphi}$ is equal to $\hat{\varphi} \overline{\hat{\varphi}}=|\widehat{\varphi}|^{2} \geq 0$. Hence, $\varphi * \widetilde{\varphi}$ is positive definite on $\mathbb{R}^{n}$. If $f$ satisfies (2.7) for all positive definite $\omega \in D\left(\mathbb{R}^{n}\right)$, then we can take $\omega=\varphi * \widetilde{\varphi}$. Thus, (1.1) holds.

Lemma 2.3. Suppose that $\varphi \in \mathcal{E}\left(\mathbb{R}^{n}\right)$ is positive definite. Then there exists a sequence $\left(\psi_{k}\right)$ of positive definite $\psi_{k} \in D\left(\mathbb{R}^{n}\right), k=1,2, \ldots$, such that $\lim _{k \rightarrow \infty} \psi_{k}=\varphi$ in the topology of $\mathcal{E}\left(\mathbb{R}^{n}\right)$.

Proof. Take any $\sigma_{1} \in D\left(\mathbb{R}^{n}\right)$ such that $\left\|\sigma_{1}\right\|_{L^{2}\left(\mathbb{R}^{n}\right)}=1$. Set $\sigma=\sigma_{1} * \widetilde{\sigma}_{1}$. Then $\sigma$ is positive definite. Hence,

$$
|\sigma(x)| \leq \sigma(0)=\left\|\sigma_{1}\right\|_{L^{2}\left(\mathbb{R}^{n}\right)}=1, \quad x \in \mathbb{R}^{n} .
$$

Now we define the function $\psi_{k}(x)$ to be $\sigma(x / k) \varphi$ for $k=1,2, \ldots$ Of course, $\psi_{k}(x) \in D\left(\mathbb{R}^{n}\right)$ and $\psi_{k}(x)$ is positive definite as a product of two positive definite functions. We recall that a sequence $\left\{\theta_{j}\right\}_{j} \in \mathcal{E}\left(\mathbb{R}^{n}\right)$ converges in $\mathcal{E}\left(\mathbb{R}^{n}\right)$ to $\theta \in$ $\mathcal{E}\left(\mathbb{R}^{n}\right)$ if and only if for every multi-index $u \in \mathbb{R}^{n}$, the sequence $\left\{D_{x}^{u} \theta_{j}\right\}_{j}$ converges uniformly to $D_{x}^{u} \theta$ on every compact subset of $\mathbb{R}^{n}$. By (2.8), it is easy to see that for any fixed multi-index $u \in \mathbb{R}^{n}$, the sequence $D_{x}^{u}[\sigma(x / k)-1], k=1,2, \ldots$, 
converges to the zero function as $k \rightarrow \infty$ uniformly on compact subsets of $\mathbb{R}^{n}$. Finally, since

we finish the proof.

$$
\varphi(x)-\psi_{k}(x)=\varphi(x)[\sigma(x / k)-1]
$$

We are now in a position to prove the necessity of Theorem 1.2.

Proof of Theorem 1.2 (Necessity).

Suppose that $\Gamma$ is a regular cone in $\mathbb{R}^{n}$ and let $y \in \Gamma$. If $t \in \mathbb{R}^{n}$, then using (2.1) and (2.2), we see that

$D_{y}^{u} K_{\Gamma}(i y-t)=\frac{\partial^{|u|}}{\partial y_{1}^{u_{1}} \ldots \partial y_{n}^{u_{n}}} K_{\Gamma}(i y-t)=(-1)^{|u|} \int_{\Gamma^{*}}\left(\xi_{1}^{u_{1}} \cdots \xi_{n}^{u_{n}}\right) e^{-(y, \xi)} e^{-i(t, \xi)} d \xi$

for each multi-index $u \in \mathbb{R}^{n}$. In particular, if $\gamma=\left(\gamma_{1}, \ldots, \gamma_{n}\right) \in \Gamma$, then for the directional derivative $D_{\gamma}$ of $K_{\Gamma}$, we have

$$
\begin{array}{r}
D_{\gamma} K_{\Gamma}(i y-t)=\left(\gamma, D_{y}\right) K_{\Gamma}(i y-t)=\sum_{s=1}^{n} \gamma_{s} \frac{\partial}{\partial y_{s}} K_{\Gamma}(i y-t) \\
=-\int_{\Gamma^{*}}\left(\sum_{s=1}^{n} \gamma_{s} \xi_{s}\right) e^{-(y, \xi)} e^{-i(t, \xi)} d \xi=-\int_{\Gamma^{*}}(\gamma, \xi) e^{-(y, \xi)} e^{-i(t, \xi)} d \xi .
\end{array}
$$

Iterating (2.9), we obtain, for an arbitrary set $\gamma^{(1)}, \ldots, \gamma^{(k)} \in \Gamma$, that

$$
\begin{array}{r}
D_{\gamma^{(1)}} D_{\gamma^{(2)}} \ldots D_{\gamma^{(k)}} K_{\Gamma}(i y-t)=(-1)^{k} \int_{\Gamma^{*}} \prod_{j=1}^{k}\left(\gamma^{(j)}, \xi\right) e^{-(y, \xi)} e^{-i(t, \xi)} d \xi \\
=(-1)^{k} \int_{\mathbb{R}^{n}}\left(\prod_{j=1}^{k}\left(\gamma^{(j)}, \xi\right) e^{-(y, \xi)} \chi_{\Gamma^{*}}(\xi)\right) e^{-i(t, \xi)} d \xi
\end{array}
$$

For fixed $y \in \Gamma$ and for $\theta \in \Gamma$, we define the function $E_{\theta}$ by

$$
E_{\theta}(\xi)=(\theta, \xi) e^{-(y, \xi)} \chi_{\Gamma^{*}}(\xi), \quad \xi \in \mathbb{R}^{n}
$$

Since $\Gamma$ is an open cone, it is easy to see that there exists $\delta=\delta(y)>0$ such that

$$
(y, \xi) \geq \delta|\xi|_{2} \quad \text { for all } \xi \in \Gamma^{*}
$$

(see, e.g., [14, p. 104]). Hence, $E_{\theta}$ is a nonnegative bounded and integrable function on $\mathbb{R}^{n}$. Note that the function

$$
\xi \rightarrow \prod_{j=1}^{k}\left(\gamma^{(j)}, \xi\right) e^{-(y, \xi)} \chi_{\Gamma^{*}}(\xi), \quad \xi \in \mathbb{R}^{n}
$$

which we used in (2.10), is equal to

$$
\prod_{j=1}^{k} E_{\gamma^{(j)}}(\xi)
$$

Hence, the function (2.11) is also nonnegative bounded and integrable on $\mathbb{R}^{n}$. Thus, applying the Bochner theorem to the right side of (2.10), we see that, for 
any fixed $y \in \Gamma$ and for any choice of $\gamma^{(1)}, \ldots, \gamma^{(k)} \in \Gamma$, the function

$$
(-1)^{k} D_{\gamma^{(1)}} D_{\gamma^{(2)}} \ldots D_{\gamma^{(k)}} K_{\Gamma}(i y-t)
$$

is continuous positive definite as a function of $t \in \mathbb{R}^{n}$. Moreover, by Lemma 2.1, the function $K_{\Gamma}(z)$ and its derivative (2.12) are analytic on $T_{\Gamma}$. Hence, (2.12) belongs to $\mathcal{E}\left(\mathbb{R}^{n}\right)$ as a function of $t$.

Suppose now that $f \in \mathcal{E}^{\prime}\left(\mathbb{R}^{n}\right)$ and that $f$ is positive definite. Using Lemma 2.3, we see that, for any fixed choice of $y \in \Gamma, \gamma^{(1)}, \ldots, \gamma^{(k)} \in \Gamma$, there exists a sequence $\left(\psi_{m}\right)$ of positive definite functions $\psi_{m} \in D\left(\mathbb{R}^{n}\right), m=1,2, \ldots$, such that

$$
\lim _{m \rightarrow \infty} \psi_{m}(t)=(-1)^{k} D_{\gamma^{(1)}} D_{\gamma^{(2)}} \ldots D_{\gamma^{(k)}} K_{\Gamma}(i y-t)
$$

in the topology of $\mathcal{E}\left(\mathbb{R}^{n}\right)$. Then by Lemma 2.2, we get

$$
\left.(-1)^{k}\left(f_{t}, D_{\gamma_{1}} D_{\gamma_{2}} \ldots D_{\gamma_{k}} K_{\Gamma}(i y-t)\right)\right)=\lim _{m \rightarrow \infty}\left(f_{t}, \psi_{m}(t)\right) \geq 0
$$

Combining this with (1.7) and (2.3), we see that

$$
(-1)^{k} D_{\gamma^{(1)}} D_{\gamma^{(2)}} \ldots D_{\gamma^{(k)}} K_{\Gamma}(f)(i y) \geq 0 .
$$

This shows that $y \rightarrow K_{\Gamma}(f)(i y)$ is a completely monotonic function on $\Gamma$. Necessity of Theorem 1.2 is proved.

We will use the following lemma (see, e.g., [3, p. 211]), which gives an analytic Cauchy representation for any distribution with compact support.

Lemma 2.4. Suppose that $\left\{\Gamma_{k}\right\}_{1}^{m}$ is a family of regular cones such that $\left\{\Gamma_{k}^{*}\right\}_{1}^{m}$ covers exactly $\mathbb{R}^{n}$. Let $y^{(k)} \in \Gamma_{k}, k=1, \ldots, m$. If $f \in \mathcal{E}^{\prime}\left(\mathbb{R}^{n}\right)$, then

$$
\lim _{\max \left\|y^{(k)}\right\|_{2} \rightarrow 0} \sum_{k=1}^{m} \int_{\mathbb{R}^{n}} K_{\Gamma_{k}}(f)\left(x+i y^{(k)}\right) \omega(x) d x=(f, \omega)
$$

for all $\omega \in D\left(\mathbb{R}^{n}\right)$.

Proof of Theorem 1.2. (Sufficiency). Let $\Lambda$ be a regular cone in $\mathbb{R}^{n}$. Let $g \in \mathcal{E}^{\prime}\left(\mathbb{R}^{n}\right)$ and suppose that the function $y \rightarrow K_{\Lambda}(g)(i y)$ is completely monotonic on $\Lambda$. We claim that for any fixed $y \in \Gamma$, the function

$$
x \rightarrow K_{\Lambda}(g)(x+i y)
$$

is continuous and positive definite on $\mathbb{R}^{n}$. Since $\Lambda$ is convex, it follows that $\Lambda$ is an additive semigroup. Fix a point $\delta \in \Lambda$. Because $\Lambda$ is open, it is easy to see that

$$
\delta+\bar{\Lambda} \subset \Lambda
$$

Define the function

$$
G(y)=K_{\Lambda}(g)(i(\delta+y)) .
$$

By (2.13), this function is well defined for all $y \in \bar{\Lambda}$. Of course, it is completely monotonic on $\Lambda$. Moreover, using (2.13), we see that $G$ is continuous on $\bar{\Lambda}$. Then (see $\left[6\right.$, p. 172]) there exists a nonnegative measure $\mu_{\delta, \Lambda}$ on $\Lambda^{*}$ such that

$$
G(y)=\int_{\Lambda^{*}} e^{-(y, \zeta)} d \mu_{\delta, \Lambda}(\zeta)
$$


for all $y \in \bar{\Lambda}$. Since $0 \in \bar{\Lambda}$ and $G$ is continuous on $\bar{\Lambda}$, then

$$
G(0)=\int_{\Lambda^{*}} d \mu_{\delta, \Lambda}(\zeta)
$$

Hence, $\mu_{\gamma, \Lambda}$ is a finite measure. Therefore, the function $G$ can be extended analytically on $T_{\Lambda}$ as the Laplace transform of $\mu_{\gamma, \Lambda}$, i.e., for $z=x+i y \in T_{\Gamma}$, we can set

$$
G(z)=\int_{\Lambda^{*}} e^{i(z, \zeta)} d \mu_{\gamma, \Lambda}(\zeta)
$$

Note that this integral converges absolutely.

By (2.14), the function $G(z)$ coincides with $K_{\Lambda}(g)(i \delta+z)$ for $z=i y, y \in \bar{\Lambda}$. We will show that this is true also for all $z \in T_{\Lambda}$. To this end, we use the following identity theorem (see e.g., [10, p.16-17]): if $H$ is an analytic function on an open connected domain $D$ in $\mathbb{C}^{n}, a \in D$, and $H(a+x)=0$ for all $x$ in a neighborhood of 0 in $\mathbb{R}^{n}$, then $H \equiv 0$ on $D$. Of course, a similar statement is valid also in the case if we replace a real neighborhood of $a$ by any imaginary neighborhood, i.e., if we have $H(a+i y)=0$ for all $y$ in a neighborhood of 0 in $\mathbb{R}^{n}$, then also $H \equiv 0$ on $D$. Now fix any $z_{0}=i y_{0} \in i \Lambda \subset T_{\Lambda}$. Then using (2.13)and (2.14), we see that $G(z)$ and $K_{\Lambda}(g)(i \gamma+z)$ coincide for all $z$ in an imaginary neighborhood $I_{z_{0}}=\left\{z=x+i y \in \mathbb{C}^{n}:\left|y-y_{0}\right|<r, x=x_{0}\right\}$ of $z_{0}$ such that $I_{z_{0}} \subset T_{\Lambda}$. Hence, $G(z)=K_{\Lambda}(g)(i \delta+z)$ for all $z \in T_{\Lambda}$. Moreover, by (2.14) and (2.15), we have that

$$
K_{\Lambda}(g)(i \delta+z)=G(z)=\int_{\Lambda^{*}} e^{i(z, \zeta)} d \mu_{\gamma, \Lambda}(\zeta)=\int_{\Lambda^{*}} e^{i(x, \zeta)} e^{-(y, \zeta)} d \mu_{\gamma, \Lambda}(\zeta)
$$

for all $z=x+i y \in T_{\Lambda}$. Using (2.16) and having the Bochner theorem for continuous positive definite functions on $\mathbb{R}^{n}$, we obtain that for any fixed $y \in \Gamma$, the functions

$$
x \rightarrow G(x+i y) \quad \text { and } \quad x \rightarrow K_{\Lambda}(g)(x+i(\delta+y))
$$

are continuous and positive definite on $\mathbb{R}^{n}$. Thus, since $\Lambda$ is open and $\delta$ is an arbitrary point of $\Lambda$, we obtain that the function $x \rightarrow K_{\Lambda}(g)(x+i y)$ also is continuous and positive definite on $\mathbb{R}^{n}$. This proves our claim.

Let $\left\{\Gamma_{k}\right\}_{k=1}^{m}$ be as in the Theorem 1.2 and suppose that for $f \in \mathcal{E}^{\prime}\left(\mathbb{R}^{n}\right)$, the functions $y \rightarrow K_{\Gamma_{k}}(f)(i y), y \in \Gamma_{k}, k=1,2, \ldots, m$ are completely monotonic. Fix $y^{(1)} \in \Gamma_{1}, \ldots, y^{(m)} \in \Gamma_{m}$, and define

$$
F(x)=\sum_{k=1}^{m} K_{\Gamma_{k}}(f)\left(x+i y^{(k)}\right)
$$

for $x \in \mathbb{R}^{n}$. We just proved that each $x \rightarrow K_{\Gamma_{k}}(f)\left(x+i y^{(k)}\right), k=1, \ldots, m$, is a continuous and positive definite function on $\mathbb{R}^{n}$. Hence, the same is still true for (2.17). If $\omega \in D\left(\mathbb{R}^{n}\right)$, then $F \cdot \omega$ is integrable on $\mathbb{R}^{n}$, and by Lemma 2.2, we have that

$$
\sum_{k=1}^{m} \int_{\mathbb{R}^{n}} K_{\Gamma_{k}}(f)\left(x+i y^{(k)}\right) \omega(x) d x=\int_{\mathbb{R}^{n}} F(x) \omega(x) d x \geq 0 .
$$


Letting now $\max \left\|y^{(k)}\right\|_{2} \rightarrow 0$. Then (2.18) this, together with Lemma 2.4, proves that $(f, \omega) \geq 0$ for all $\omega \in D\left(\mathbb{R}^{n}\right)$. Thus, $f$ is a positive definite distribution.

Acknowledgement. The author thanks the referee for pointing out several mistakes and making a few other remarks which improved the exposition. This research was funded by a grant (No. MIP-053/2012) from the Research Council of Lithuania.

\section{REFERENCES}

1. P. Blanchard and E. Brüning, Mathematical Methods in Physics. Distributions, Hilbert space Operators and Variational Methods. Progress in Mathematical Physics, Birkhäuser, Boston-Basel-Berlin, 2003.

2. S. Bochner, Harmonic analysis and the theory of probability, Dover Publications, New York, 2005.

3. R.D. Carmichael and D. Mitrovič, Distributions and analytic functions, Pitman Research Notes in Mathematics Series, vol. 206, Longman Scientific \& Technical, Harlow; copublished in the United States with John Wiley \& Sons, Inc., New York, 1989.

4. J. Chung, S.-Y. Chung and D. Kim D, Positive definite hyperfunctions, Nagoya Math. J. 140 (1995), 139-149.

5. E. Hewitt and K.A. Ross, Abstract Harmonic Analysis, vol. 2, Springer, Berlin-Heidelberg, 1997.

6. F. Hirsch, Familles résolvantes, générateurs, cogénérateurs, potentiels, Ann. Inst. Fourier 22 (1972), no. 1, 89-210.

7. A.W. Knapp, Advanced real analysis. Along with a companion volume Basic real analysis. Cornerstones. Birkhäuser Boston, Inc., Boston, MA, 2005.

8. E. Lukacs, Characteristic Functions. 2nd ed., Hafner Publishing Co., New York, 1970.

9. S. Norvidas, A note on positive definite distributions, Indag. Math. 24 (2013), no. 3, 505-517.

10. R.M. Range, Holomorphic functions and integral representations in several complex variables. Graduate Texts in Mathematics, 108. Springer-Verlag, New York, 1986.

11. H.-J. Rossberg, Positive definite probability densities and probability distributios, J. Math. Sci. 76, (1995) no. 1, 2181-2197.

12. Z. Sasvári, Multivariate Characteristic and Correlation Functions, De Gruyter, Berlin, 2013.

13. O.J. Staffans, Positive definite measures with applications to a Volterra equation, Trans. Amer. Soc. 218 (1976), 219-237.

14. E.M. Stein and G. Weiss, Introduction to Fourier analysis on Euclidean spaces. Princeton Mathematical Series, No. 32. Princeton University Press, Princeton, N.J., 1971.

15. H.G Tillmann, Distributionen als Randvertailungen analytischer Funktionen. II., Math. Z. 76 (1961), 5-21.

16. V.S. Vladimirov, Methods of the Theory of Generalized Functions, Taylor \& Francis, London, 2002.

Vilnius University Institute of Mathematics and Informatics, Akademijos 4, LT-08663 VilniUs, LithuANia;

Mykolas Romeris University, Ateities 20, LT-08303 Vilnius, Lithuania.

E-mail address: norvidas@gmail.com 